\title{
Certain fundamental properties of generalized natural transform in generalized spaces
}

\author{
Shrideh Khalaf Al-Omari ${ }^{*}$ (D) and Serkan Araci $^{2}$ (D)
}

"Correspondence:

shridehalomari@bau.edu.jo;

s.k.q.alomari@fet.edu.jo

'Department of Physics and Basic

Sciences, Faculty of Engineering

Technology, Al-Balqa Applied

University, 11134 Amman, Jordan

Full list of author information is

available at the end of the article

\section{Springer}

\begin{abstract}
This paper considers the definition and the properties of the generalized natural transform on sets of generalized functions. Convolution products, convolution theorems, and spaces of Boehmians are described in a form of auxiliary results. The constructed spaces of Boehmians are achieved and fulfilled by pursuing a deep analysis on a set of delta sequences and axioms which have mitigated the construction of the generalized spaces. Such results are exploited in emphasizing the virtual definition of the generalized natural transform on the addressed sets of Boehmians. The constructed spaces, inspired from their general nature, generalize the space of integrable functions of Srivastava et al. (Acta Math. Sci. 35B:1386-1400, 2015) and, subsequently, the extended operator with its good qualitative behavior generalizes the classical natural transform. Various continuous embeddings of potential interests are introduced and discussed between the space of integrable functions and the space of integrable Boehmians. On another aspect as well, several characteristics of the extended operator and its inversion formula are discussed.
\end{abstract}

MSC: Primary 54C40; 14E20; secondary 46E25; 20C20

Keywords: Natural transform; Generalized natural transform; Boehmian; UltraBoehmian; Sumudu transform; Laplace transform

\section{Introduction and preliminaries}

The integral transform operators have attained their popularity due to their wide range of applications in various fields of science and engineering as, in most of cases, the physical phenomenon is converted into ordinary and partial differential equations. Along with interesting groups of integral transforms arising in literature, the natural transform NT was introduced by Khan and Khan [2] and renamed recently as the $N$-transform [3-6]. In addition to the shift and change of scale properties of the NT, the authors of [5] solved the unsteady fluid flow problem over a plane wall and highlighted that the transform converges to the Laplace and Sumudu transforms. Later, Belgacem et al. [3] defined the inverse natural transform formula and studied some properties and applications on Maxwell's equation. When the real sectionwise continuous function $\varphi(t)>0, \varphi(t)=0$ for $t<0$ is of exponential

(c) The Author(s) 2021. This article is licensed under a Creative Commons Attribution 4.0 International License, which permits use sharing, adaptation, distribution and reproduction in any medium or format, as long as you give appropriate credit to the original author(s) and the source, provide a link to the Creative Commons licence, and indicate if changes were made. The images or other third party material in this article are included in the article's Creative Commons licence, unless indicated otherwise in a credit line to the material. If material is not included in the article's Creative Commons licence and your intended use is not permitted by statutory regulation or exceeds the permitted use, you will need to obtain permission directly from the copyright holder. To view a copy of this licence, visit http://creativecommons.org/licenses/by/4.0/. 
order defined on $A$, where

$$
A=\left\{\varphi(t)\left|\exists M, \epsilon_{1}, \epsilon_{2}>0,\right| \varphi(t) \mid<M e^{\frac{t}{\epsilon_{j}}} \text {,if } t \in(-1)^{j} \times[0, \infty)\right\},
$$

the natural transform NT is given by [2]

$$
N(\varphi)(u, v)=\int_{0}^{\infty} e^{-u t} \varphi(v t) d t, \quad u>0, v>0 .
$$

For $\alpha \in \mathbb{C} ; \operatorname{Re}(\alpha) \geq 0$ and $k \in \mathbb{Z}_{+}$, the generalized natural transform GNT or the $M_{\alpha, k}$ transform of a function $\varphi$ was proclaimed as $[1,(1.1)]$

$$
M_{\alpha, k}(\varphi)(u, v)=\int_{0}^{\infty} \varphi(v t) \frac{e^{-u t}}{\left(t^{k}+v^{k}\right)^{\alpha}} d t
$$

provided the integral part exists. The integral part of the preceding equation can be indeed motivated to yield

$$
M_{\alpha, k}(\varphi)(u, v)=v^{-k \alpha-1} \int_{0}^{\infty} \varphi(t) \frac{e^{-\frac{u}{v} t}}{\left(t^{k}+v^{k}\right)^{\alpha}} d t
$$

where $u$ and $v$ are the transform variables. The GNT transform corresponds to the NT for $\alpha=0$ [8] and to the Stieltjes transform for $u=0$ [9]. On top of that, it corresponds to the Laplace transform [10]

$$
L(\varphi)(v)=\int_{0}^{\infty} e^{-v t} \varphi(t) d t
$$

for $\alpha=0$ and $u=1$ and to the Sumudu transform [7]

$$
S(\varphi)(u)=\int_{0}^{\infty} e^{-t} \varphi(u t) d t, \quad u \in\left(-\epsilon_{1}, \epsilon_{2}\right)
$$

for $\alpha=0$ and $v=1$, see, e.g., [7, 11-17]. The Parseval type theorem of the GNT transform is given by [1, Theorem 3.1]

$$
\int_{0}^{\infty} \frac{\varphi(v u)}{\left(u^{k}+v^{k}\right)^{\alpha_{1}}} M_{\alpha_{2}, k}(g)(t, v) d t=\int_{0}^{\infty} \frac{g(v t)}{\left(t^{k}+v^{k}\right)^{\alpha_{2}}} M_{\alpha_{1}, k}(\varphi)(t, v) d t
$$

The scaling property of the GNT transform for $\beta>0$ is given by $[1,(2.4)]$

$$
M_{\alpha, k}\left(\varphi\left(\beta^{2} t\right)\right)(u, v)=\beta^{k \alpha-1} M_{\alpha, k}(\varphi(t))\left(\frac{u}{\beta}, \beta v\right) .
$$

Due to [1, Theorem 2.1], the GNT transform of $\varphi$ exists for all $0<v<\mu$ and $\operatorname{Re}(u)>$ $\frac{\mu}{\beta}$, where the function $\varphi$ is either continuous or piecewise continuous on $[0, \infty)$ and for certain given $K, T, \beta>0$,

$$
|\varphi(t)| \leq K t^{k R e(\alpha)} e^{t / \beta} \quad \text { for all } t>T .
$$


Further, it converges uniformly with respect to the transform variable $u$ provided $\operatorname{Re}(u) \geq$ $\alpha>\frac{\mu}{\beta}$. The inversion of the GNT transform is defined by

$$
\varphi(t)=\left(\frac{t^{m}}{v^{m}}+v^{m}\right)^{\alpha} L^{-1}\left(M_{\alpha, k}(\varphi(t))(u, v)\right)\left(\frac{t}{v}\right), \quad v \in(0, \mu)
$$

provided that the involved integral converges absolutely, where $L^{-1}$ is the inverse Laplace transform operator. The Mellin-type convolution product of two integrable functions $\varphi$ and $g$ is defined by [18]

$$
(\varphi \ominus g)(y)=\int_{0}^{\infty} \varphi(x) g\left(y x^{-1}\right) \frac{d x}{x}
$$

when the integral part exists. Consequently, the natural properties of this convolution product are due to [19] given by

$$
\begin{aligned}
& \varphi \ominus g=g \ominus \varphi, \quad\left(\varphi \ominus g_{1}\right) \ominus g_{2}=\varphi \ominus\left(g_{1} \ominus g_{2}\right) \quad \text { and } \\
& \varphi \ominus\left(g_{1}+g_{2}\right)=\varphi \ominus g_{1}+\varphi \ominus g_{2} .
\end{aligned}
$$

Evaluations of the GNT transform of various special functions, polynomials, and derivatives are computed in the above citations. In this article we derive a convolution theorem and establish sets of generalized functions for the considered GNT transform. In Sect. 1, we have already reviewed certain definitions and preliminaries from literature. In Sect. 2, we derive the convolution theorem and provide auxiliary results to facilitate our next investigations. In Sect. 3, we prove axioms and determine spaces of Boehmians and give the extension of the generalized natural transform to the Boehmian spaces. In Sect. 4, we derive some characteristics of the transform in a sense of generalized functions.

\section{Convolution theorem and auxiliary results}

To proceed in this study, we denote by $L^{1}\left(\mathbb{R}_{+}^{2}\right)$ the Lebesgue space of integrable functions over $\mathbb{R}_{+}^{2}=\mathbb{R}_{+} \times \mathbb{R}_{+}, \mathbb{R}_{+}=(0, \infty)$ and by $C^{\infty}\left(\mathbb{R}_{+}\right)$the Schwartz space of smooth functions of compact supports over $\mathbb{R}_{+}$. On the basis of the convolution product $\ominus$, we present a convolution formula that is very useful in the sequel.

Definition 1 Let $k \in \mathbb{Z}_{+}$and $\alpha \in \mathbb{C}$ such that $\operatorname{Re}(\alpha) \geq 0$. We denote by $\oplus$ the integral equation

$$
(\psi \oplus \varphi)(u, v)=\int_{0}^{\infty} \varphi(x) \psi(\sqrt{x} u, \sqrt{x} v) \frac{d_{x}}{x^{k \alpha}}
$$

provided the right-hand side of the above equation exists for every $u>0$ and $v>0$.

By taking into account Eq. (4) and Eq. (6), we derive a convolution theorem as follows.

Theorem 2 Let $\varphi \in L^{1}\left(\mathbb{R}_{+}\right), k \in \mathbb{Z}_{+}$, and $\alpha \in \mathbb{C}$ such that $\operatorname{Re}(\alpha) \geq 0$. Then we have

$$
M_{\alpha, k}(\varphi \ominus g)=\varphi \oplus M_{\alpha, k} g
$$

for every $g \in C^{\infty}\left(\mathbb{R}_{+}\right)$. 
Proof Let $\varphi \in L^{1}\left(\mathbb{R}_{+}\right), k \in \mathbb{Z}_{+}$, and $\alpha \in \mathbb{C}$ such that $\operatorname{Re}(\alpha) \geq 0$. Then invoking Eq. (4), Eq. (1) gives

$$
M_{\alpha, k}(\varphi \ominus g)(u, v)=v^{-k \alpha-1} \int_{0}^{\infty} \frac{e^{-\frac{u}{v} y}}{\left(y^{k}+v^{k}\right)^{\alpha}} \int_{0}^{\infty} \varphi(x) g\left(y x^{-1}\right) \frac{d x}{x} d y .
$$

Therefore, Fubini's theorem leads to

$$
M_{\alpha, k}(\varphi \ominus g)(u, v)=v^{-k \alpha-1} \int_{0}^{\infty} \varphi(x) \int_{0}^{\infty} \frac{e^{-\frac{u}{v} y}}{\left(y^{k}+v^{k}\right)^{\alpha}} g\left(y x^{-1}\right) d y \frac{d x}{x}
$$

By using the change of variables $y=z x$, along with simple computations, we get

$$
\begin{aligned}
M_{\alpha, k}(\varphi \ominus g)(u, v) & =v^{-k \alpha-1} \int_{0}^{\infty} \varphi(x) \int_{0}^{\infty} \frac{e^{-\frac{u}{v} x z}}{\left((z x)^{k}+v^{k}\right)^{\alpha}} g(z) d z d x \\
\text { i.e. } & =v^{-k \alpha-1} \int_{0}^{\infty} \varphi(x) \int_{0}^{\infty} \frac{e^{-\frac{u}{v} x z}}{x^{k \alpha}\left(z^{k}+\left(\frac{v}{\sqrt{x}}\right)^{k}\right)^{\alpha}} g(z) d z d x \\
\text { i.e. } & =v^{-k \alpha-1} \int_{0}^{\infty} \varphi(x) \int_{0}^{\infty} \frac{e^{-\frac{u \sqrt{x}}{v}} \frac{v}{\sqrt{x}}}{\left(z^{k}+\left(\frac{v}{\sqrt{x}}\right)^{k}\right)^{\alpha}} g(z) d z \frac{d x}{x^{k \alpha}} \\
\text { i.e. } & =\int_{0}^{\infty} \varphi(x)\left(M_{\alpha, k} g\right)(\sqrt{x} u, \sqrt{x} v) \frac{d x}{x^{k \alpha}} .
\end{aligned}
$$

This completes the proof of the theorem.

By $\Delta$ we denote the subset of the Schwartz space $C^{\infty}\left(\mathbb{R}_{+}\right)$of delta sequences $\left\{\delta_{0}, \delta_{1}, \ldots\right.$, $\left.\delta_{n}, \ldots\right\}$ such that Eq. (8) to Eq. (10) hold

$$
\begin{aligned}
& \int_{0}^{\infty} \delta_{n}(x) d x=1, \quad \forall n \in \mathbb{N}, \\
& \int_{0}^{\infty}\left\|\delta_{n}(x)\right\|_{L^{1}\left(\mathbb{R}_{+}^{2}\right)} d x \leq c, \quad \text { for some constant } c \text { and all } n \in \mathbb{N}, \\
& \lim _{n \rightarrow \infty} \int_{|x|>\varepsilon}|x|^{k}\left\|\delta_{n}(x)\right\|_{L^{1}\left(\mathbb{R}_{+}^{2}\right)} d x=0, \quad \forall k \in \mathbb{N}, \varepsilon>0 .
\end{aligned}
$$

Theorem 3 Let $U \in L^{1}\left(\mathbb{R}_{+}^{2}\right)$ and $\phi_{1}, \phi_{2} \in C^{\infty}\left(\mathbb{R}_{+}\right)$. Then we have

$$
U \oplus\left(\phi_{1} \ominus \phi_{2}\right)=\left(U \oplus \phi_{1}\right) \oplus \phi_{2} \quad \text { in } L^{1}\left(\mathbb{R}_{+}^{2}\right)
$$

Proof Let $U \in L^{1}\left(\mathbb{R}_{+}^{2}\right)$ and $\phi_{1}, \phi_{2} \in C^{\infty}\left(\mathbb{R}_{+}\right)$be given arbitrary. Then, by applying Eq. (6) and Eq. (4), we assert

$$
\begin{aligned}
\left(U \oplus\left(\phi_{1} \ominus \phi_{2}\right)\right)(u, v) & =\int_{0}^{\infty} U(\sqrt{x} u, \sqrt{x} v)\left(\phi_{1} \ominus \phi_{2}\right)(x) \frac{d x}{x^{k \alpha}} \\
& =\int_{0}^{\infty} \phi_{2}(t) \int_{0}^{\infty} U(\sqrt{x} u, \sqrt{x} v) \phi_{1}\left(x t^{-1}\right) \frac{d x}{x^{k \alpha}} \frac{d t}{t}
\end{aligned}
$$




$$
\begin{aligned}
& =\int_{0}^{\infty} \phi_{2}(t) \int_{0}^{\infty} U(\sqrt{t z} u, \sqrt{t z} v) \phi_{1}(z) \frac{d z}{z^{k \alpha}} \frac{d t}{t^{k \alpha}} \\
& =\int_{0}^{\infty} \phi_{2}(t)\left(U \oplus \phi_{1}\right)(\sqrt{t} u, \sqrt{t} v) \frac{d t}{t^{k \alpha}} .
\end{aligned}
$$

Hence, we have obtained

$$
\left(U \oplus\left(\phi_{1} \ominus \phi_{2}\right)\right)(u, v)=\left(\left(U \oplus \phi_{1}\right) \oplus \phi_{2}\right)(u, v) .
$$

This proves the first part. To complete the inclusion part of the theorem, we show that

$$
U \oplus \phi \in L^{1}\left(\mathbb{R}_{+}^{2}\right) \quad \text { for } U \in L^{1}\left(\mathbb{R}_{+}^{2}\right) \text { and } \phi \in C^{\infty}\left(\mathbb{R}_{+}\right)
$$

Indeed, from Eq. (6) and Fubini's theorem, we obtain

$$
\begin{aligned}
\|U \oplus \phi\|_{L^{1}\left(\mathbb{R}_{+}^{2}\right)} & =\int_{\mathbb{R}_{+}^{2}}|(U \oplus \phi)(u, v)| d(u, v) \\
& \leq \int_{\mathbb{R}_{+}^{2}} \int_{\mathbb{R}_{+}}|U(\sqrt{x} u, \sqrt{x} v)||\phi(x)| d x d(u, v) .
\end{aligned}
$$

The definition of the norm $\|\cdot\|_{L^{1}\left(\mathbb{R}_{+}^{2}\right)}$ of $L^{1}\left(\mathbb{R}_{+}^{2}\right)$ indeed implies that

$$
\|U \oplus \phi\|_{L^{1}\left(\mathbb{R}_{+}^{2}\right)} \leq\|U\|_{L^{1}\left(\mathbb{R}_{+}^{2}\right)} \int_{\mathbb{R}_{+}}|\phi(x)| d x .
$$

Therefore, the right-hand side of Eq. (12) is bounded by the compactness of the support of $\phi$. Hence, our theorem is completely proved.

\section{The spaces $B_{1}$ and $B_{2}$}

To proceed in the construction of the abstract Boehmian space, we demand two sets, say $G$ and $S$, and two operations, say $\star$ and $*$, where $G$ is a topological vector space, $S$ is a subspace of $G$ and, for $\alpha, \beta \in G$ and $x, y \in S$, the operation $\star: G \times S \rightarrow G$ and $*$ satisfy the axioms: $x * y=y * x \in S,(\alpha \star x) \star y=\alpha \star(x * y),(\alpha+\beta) \star x=\alpha \star x+\beta \star x$; and as $\alpha_{n} \rightarrow \alpha$ in $G$, we have $\alpha_{n} \star y \rightarrow \alpha \star y$ for sufficiently large values of $n$. Besides, there should be a collection $\Delta$ of sequences in $S$ such that:

(i) If $\left\{y_{1}, y_{2}, \ldots, y_{n}, \ldots\right\},\left\{x_{1}, x_{2}, \ldots, x_{n}, \ldots\right\} \in \Delta$, then $\left\{x_{1} * y_{1}, x_{2} * y_{2}, \ldots, x_{n} * y_{n}, \ldots\right\} \in \Delta$.

(ii) If $\alpha \in G$ and $\left\{y_{1}, y_{2}, \ldots, y_{n}, \ldots\right\} \in \Delta$, then $\alpha \star y_{n} \rightarrow \alpha$ in $G$ as $n \rightarrow \infty$.

Let $\left\{\alpha_{1}, \alpha_{2}, \ldots, \alpha_{n}, \ldots\right\} \in G$ and $\left\{y_{1}, y_{2}, \ldots, y_{n}, \ldots\right\} \in \Delta$, then by $A$ we denote the collection of all pairs of sequences $\left(\alpha_{n}, y_{n}\right)$ such that $\alpha_{n} \star y_{m}=\alpha_{m} \star y_{n}, m, n \in \mathbb{N}$. Each element of $A$ is said to be a quotient of sequences and is denoted by $\frac{\alpha_{n}}{y_{n}}$. We define a relation $\sim$ on $A$ by $\alpha_{n} / y_{n} \sim \beta_{n} / x_{n}$ if

$$
\alpha_{n} \star x_{m}=\beta_{m} \star y_{n}, \quad \forall m, n \in \mathbb{N} .
$$

The relation $\sim$ is an equivalence relation on $A$ and decomposes it into disjoint equivalence classes. Each equivalence class is said to be a Boehmian. Every Boehmian is de- 
noted by $X_{\alpha_{n}}=\left(\frac{\alpha_{n}}{y_{n}}\right)$. The collection of all Boehmians is, for more convenience, denoted by $B(G, S, \star, \Delta)$ or $B$. Every element $\alpha$ of $G$ is identified uniquely as a member of $B$ by $\left(\frac{\alpha \star y_{n}}{y_{n}}\right)$ where $\left(y_{n}\right) \in \Delta$. The space $B$ is a vector space with

$$
\left(\frac{\alpha_{n}}{y_{n}}\right)+\left(\frac{\beta_{n}}{x_{n}}\right)=\left(\frac{\alpha_{n} \star x_{n}+\beta_{n} \star x_{n}}{y_{n} * x_{n}}\right) \quad \text { and } \quad \mu\left(\frac{\alpha_{n}}{y_{n}}\right)=\left(\frac{\mu \alpha_{n}}{y_{n}}\right), \quad \mu \in \mathbb{C} .
$$

In what follows, we construct the Boehmian spaces $B_{1} \approx B\left(L^{1}\left(\mathbb{R}_{+}\right),\left(C^{\infty}\left(\mathbb{R}_{+}\right), \Delta, \ominus\right), \ominus\right)$ and $B_{2} \approx B\left(L^{1}\left(\mathbb{R}_{+}^{2}\right),\left(C^{\infty}\left(\mathbb{R}_{+}\right), \Delta, \ominus\right), \oplus\right)$ with the products $\ominus$ (to act as $*$ ) and $\oplus$ (to act as $\star$ ) that seem to comply with the delta sequences and the operator $M_{\alpha, k}$. We refer to $[7,14$, $15,20-34]$ for an outright description and full details of abstract constructions of various Boehmian spaces and transform operators.

However, we provide several systematic hypotheses to generate the space $B_{2}$ of Boehmians. The following theorem includes a straightforward proof alluded to a simple integral calculus. Hence it has been detailed.

Theorem 4 Let $U_{n}, U, U_{1}, U_{2} \in L^{1}\left(\mathbb{R}_{+}^{2}\right)$ and $\phi \in C^{\infty}\left(\mathbb{R}_{+}\right)$. Then we have

$$
\left(U_{1}+U_{2}\right) \oplus \phi=U_{1} \oplus \phi+U_{2} \oplus \phi, \quad(\zeta U) \oplus \phi=\zeta(U \oplus \phi),
$$

where $\zeta \in C$, and, if $U_{n} \rightarrow U$ in $L^{1}\left(\mathbb{R}_{+}^{2}\right)$, then

$$
U_{n} \oplus \phi \rightarrow U \oplus \phi
$$

as $n \rightarrow \infty$ in $L^{1}\left(\mathbb{R}_{+}^{2}\right)$.

Theorem 5 If $U \in L^{1}\left(\mathbb{R}_{+}^{2}\right)$ and $\left(\delta_{n}\right) \in \Delta$, then $U \oplus \delta_{n} \rightarrow U$ as $n \rightarrow \infty$.

Proof By the property $\int_{-\infty}^{\infty} \delta_{n}=1$ of delta sequences and the definition of the norm $\|\cdot\|_{L^{1}\left(\mathbb{R}_{+}^{2}\right)}$ together with the facts that $\delta_{n} \in C^{\infty}\left(\mathbb{R}_{+}\right)$and $\operatorname{supp} \delta_{n} \subset\left(\alpha_{n}, \beta_{n}\right)$, for all $n \in \mathbb{N}$, we write

$$
\begin{aligned}
& \left\|U \oplus \delta_{n}-U\right\|_{L^{1}\left(\mathbb{R}_{+}^{2}\right)} \\
& =\int_{\mathbb{R}_{+}^{2}}\left|\int_{\mathbb{R}_{+}} U(\sqrt{x} u, \sqrt{x} v) \delta_{n}(x) \frac{d x}{x^{k \alpha}}-U(u, v)\right| d(u, v) \\
& =\int_{\mathbb{R}_{+}^{2}}\left|\int_{\mathbb{R}_{+}} U(\sqrt{x} u, \sqrt{x} v) \delta_{n}(x) \frac{d_{x}}{x^{k \alpha}}-U(u, v) \int_{\mathbb{R}_{+}} \delta_{n}(x) d x\right| d(u, v) \\
& \leq \int_{\alpha_{n}}^{\beta_{n}} \int_{\mathbb{R}_{+}^{2}}\left|\frac{U(\sqrt{x} u, \sqrt{x} v)}{x^{k \alpha}}-U(u, v)\right|\left|\delta_{n}(x)\right| d x d(u, v) .
\end{aligned}
$$

By applying certain favorable computations and considering the facts

$$
\begin{aligned}
& \left|\frac{U(\sqrt{x} u, \sqrt{x} v)}{x^{k \alpha}}-U(u, v)\right| \leq 2\|U\|_{L^{1}\left(\mathbb{R}_{+}^{2}\right)} \text { and } \\
& \left|\delta_{n}(x)\right| \leq A \text { for some real number } A,
\end{aligned}
$$


we end up with

$$
\left\|U \oplus \delta_{n}-U\right\|_{L^{1}\left(\mathbb{R}_{+}^{2}\right)} \leq 2 A\|U\|_{L^{1}\left(\mathbb{R}_{+}^{2}\right)}\left(\beta_{n}-\alpha_{n}\right) \rightarrow 0 \quad \text { as } n \rightarrow \infty .
$$

The proof is therefore ended.

The trustworthy conclusion which can be drawn from Theorems 3,4 , and 5 is the presence of the space $B_{2}$ as a Boehmian space. A Boehmian in $B_{2}$ is defined as $X_{\varphi_{n}}=\left(\frac{\varphi_{n}}{\delta_{n}}\right)$. In $B_{2}$, if $X_{\varphi_{n}}=\left(\frac{\varphi_{n}}{\delta_{n}}\right)$ and $X_{g_{n}}=\left(\frac{g_{n}}{\varepsilon_{n}}\right)$ are two Boehmians, then typically we define

$$
X_{\varphi_{n}}+X_{g_{n}}=\left(\frac{\varphi_{n} \oplus \delta_{n}+g_{n} \oplus \delta_{n}}{\delta_{n} \ominus \varepsilon_{n}}\right), \quad \beta X_{\varphi_{n}}=\left(\frac{\beta \varphi_{n}}{\delta_{n}}\right) \quad \text { for all } \beta \in \mathbb{C}
$$

Also, for $\alpha \in \mathbb{R}$ and $\omega \in L^{1}\left(\mathbb{R}_{+}^{2}\right)$, we resp. define $\oplus$, the differentiation $D^{\alpha}$, and the extension of $\oplus$ to $B_{2} \oplus L^{1}\left(\mathbb{R}_{+}^{2}\right)$ in $B_{2}$ as

$$
X_{\varphi_{n}} \oplus X_{g_{n}}=\left(\frac{\varphi_{n} \oplus g_{n}}{\delta_{n} \oplus \varepsilon_{n}}\right), \quad D^{\alpha} X_{\varphi_{n}}=\left(\frac{D^{\alpha} \varphi_{n}}{\delta_{n}}\right), \quad \text { and } \quad X_{\varphi_{n}} \oplus \omega=\frac{\varphi_{n} \oplus \omega}{\delta_{n}} .
$$

Definition 6 Let $\beta_{n}, \beta \in B_{2}, n=1,2,3, \ldots$ Then the sequence $\left\{\beta_{1}, \beta_{2}, \ldots, \beta_{n}, \ldots\right\}$ is $\delta$ convergent to $\beta$, denoted by $\delta-\lim _{n \rightarrow \infty} \beta_{n}=\beta\left(\beta_{n} \stackrel{\delta}{\rightarrow} \beta\right)$, provided there can be found a delta sequence $\left(\delta_{n}\right)$ such that

- $\left(\beta_{n} \oplus \delta_{k}\right)$ and $\left(\beta \oplus \delta_{k}\right) \in L^{1}\left(\mathbb{R}_{+}^{2}\right)$ for all $n, k \in \mathbb{N}$,

- $\lim _{n \rightarrow \infty} \beta_{n} \oplus \delta_{k}=\beta \oplus \delta_{k}$ in $L^{1}\left(\mathbb{R}_{+}^{2}\right)$ for all $k \in \mathbb{N}$.

Or, equivalently, $\delta-\lim _{n \rightarrow \infty} \beta_{n}=\beta$ if and only if there are $\varphi_{n, k}, \varphi_{k} \in L^{1}\left(\mathbb{R}_{+}^{2}\right)$ and $\left(\delta_{k}\right) \in \Delta$ such that

- $\beta_{n}=\left(\frac{\varphi_{n, k}}{\delta_{k}}\right), \beta=\left(\frac{\varphi_{k}}{\delta_{k}}\right)$,

- to every $k \in \mathbb{N}$, we havelim ${ }_{n \rightarrow \infty} \varphi_{n, k}=\varphi_{k}$ in $L^{1}\left(\mathbb{R}_{+}^{2}\right)$.

Definition 7 Let $\beta_{n}, \beta \in B_{2}$ for $n=1,2,3, \ldots$ Then the sequence $\left\{\beta_{1}, \beta_{2}, \ldots, \beta_{n}, \ldots\right\}$ is $\Delta$ convergent to $\beta$, denoted by $\Delta-\lim _{n \rightarrow \infty} \beta_{n}=\beta\left(\beta_{n} \stackrel{\Delta}{\rightarrow} \beta\right)$, provided there can be found a delta sequence $\left\{\delta_{1}, \delta_{2}, \ldots, \delta_{n}, \ldots\right\}$ such that

- $\left(\beta_{n}-\beta\right) \oplus \delta_{n} \in L^{1}\left(\mathbb{R}_{+}^{2}\right)(\forall n \in \mathbb{N})$

- $\lim _{n \rightarrow \infty}\left(\beta_{n}-\beta\right) \oplus \delta_{n}=0$ in $L^{1}\left(\mathbb{R}_{+}^{2}\right)$.

Similarly, for $U_{n}, U, U_{1}, U_{2} \in L^{1}\left(\mathbb{R}_{+}\right)$and $\phi \in C^{\infty}\left(\mathbb{R}_{+}\right)$, we can easily check the construction of the space $B_{1}$ by using the familiar properties of the Mellin type convolution (see Eq. (5)), which are $U \ominus \phi=\phi \ominus U$ and $\left(U \ominus \phi_{1}\right) \ominus \phi_{2}=U \ominus\left(\phi_{1} \ominus \phi_{2}\right)$ for $U \in L^{1}\left(\mathbb{R}_{+}\right)$and $\phi_{1}, \phi_{2} \in$ $C^{\infty}\left(\mathbb{R}_{+}\right)$, and applying analogous techniques in proving the axioms:

(i) $\left(U_{1}+U_{2}\right) \ominus \phi=U_{1} \ominus \phi+U_{2} \ominus \phi$.

(ii) $(\zeta U) \ominus \phi=\zeta(U \ominus \phi)$, where $\zeta \in \mathbb{C}$.

(iii) If $U_{n} \rightarrow U$ in $L^{1}\left(\mathbb{R}_{+}\right)$, then $U_{n} \ominus \phi \rightarrow U \ominus \phi$ as $n \rightarrow \infty$ in $L^{1}\left(\mathbb{R}_{+}\right)$.

(iv) If $U \in L^{1}\left(\mathbb{R}_{+}\right)$and $\left(\delta_{n}\right) \in \Delta$, then $U \ominus \delta_{n} \rightarrow U$ as $n \rightarrow \infty$.

Operations on $B_{1}$ can be stated as they have already been defined on the space $B_{2}$. Therefore, in $B_{1}$, if $X_{U_{n}}=\left(\frac{U_{n}}{\delta_{n}}\right)$ and $X_{V_{n}}=\left(\frac{V_{n}}{\varepsilon_{n}}\right)$ are two Boehmians, then we define

$$
X_{U_{n}}+X_{V_{n}}=\left(\frac{U_{n} \ominus \delta_{n}+V_{n} \ominus \delta_{n}}{\delta_{n} \ominus \varepsilon_{n}}\right), \quad \beta X_{U_{n}}=\left(\frac{\beta U_{n}}{\delta_{n}}\right) \quad \text { for all } \beta \in \mathbb{C}
$$


Also, for $\alpha \in \mathbb{R}$ and $U \in L^{1}\left(\mathbb{R}_{+}\right)$, we resp. define the application of $\ominus$ to Boehmians, the differentiation $D^{\alpha}$, and the extension of $\ominus$ to $B_{1} \ominus L^{1}\left(\mathbb{R}_{+}\right)$in $B_{1}$ as

$$
X_{U_{n}} \ominus X_{V_{n}}=\left(\frac{U_{n} \ominus V_{n}}{\delta_{n} \ominus \varepsilon_{n}}\right), \quad D^{\alpha} X_{U_{n}}=\left(\frac{D^{\alpha} U_{n}}{\delta_{n}}\right), \quad \text { and } \quad X_{U_{n}} \ominus U=\frac{U_{n} \ominus U}{\delta_{n}} .
$$

Hence we have the following definition.

Definition 8 Let $\left(\frac{U_{n}}{\delta_{n}}\right) \in B_{1}$, then we define the generalized $M_{\alpha, k}$ of $X_{U_{n}}=\left(\frac{U_{n}}{\delta_{n}}\right)$ as

$$
\bar{M}_{\alpha, k}\left(\frac{U_{n}}{\delta_{n}}\right)=\bar{M}_{\alpha, k} X_{U_{n}}=\left(\frac{M_{\alpha, k} U_{n}}{\delta_{n}}\right) .
$$

By the fact that $M_{\alpha, k} U_{n} \in L^{1}\left(\mathbb{R}_{+}^{2}\right)$, the formula in the above equation is well-defined.

Remark 9 Let $\left\{\Omega_{1}, \Omega_{2}, \ldots, \Omega_{n}, \ldots\right\} \in \Delta$ and $U \in L^{1}\left(\mathbb{R}_{+}^{2}\right), U=M_{\alpha, k} \psi$ for some fixed $\psi \in$ $L^{1}\left(\mathbb{R}_{+}^{2}\right)$, then we have:

(i) If $X_{\tilde{\psi}}=\left(\frac{\psi \ominus \Omega_{n}}{\Omega_{n}}\right)$, then the mapping $\psi \rightarrow X_{\tilde{\psi}}$ from $L^{1}\left(\mathbb{R}_{+}\right)$into $B_{1}$ is an injective.

(ii) If $Y_{\tilde{U}}=\left(\frac{U \oplus \Omega_{n}}{\Omega_{n}}\right)$, then the mapping $U \rightarrow Y_{\tilde{U}}$ from $L^{1}\left(\mathbb{R}_{+}^{2}\right)$ into $B_{2}$ is an injective.

From Remark 9 , it may be said that $L^{1}\left(\mathbb{R}_{+}\right)\left(\right.$resp. $\left.L^{1}\left(\mathbb{R}_{+}^{2}\right)\right)$ can be identified as subspaces of $B_{1}$ (resp. $\left.B_{2}\right)$.

\section{Remark 10}

(i) Let $\left(\psi_{n}\right) \in \Delta$. Then, if $f_{n} \rightarrow f$ in $L^{1}\left(\mathbb{R}_{+}\right)$as $n \rightarrow \infty$, then for all $k \in \mathbb{N}$,

$$
f_{n} \ominus \psi_{k} \rightarrow f \ominus \psi_{k} \quad \text { as } n \rightarrow \infty
$$

(ii) Let $\left(\Omega_{n}\right) \in \Delta$. Then, if $U_{n} \rightarrow U$ in $L^{1}\left(\mathbb{R}_{+}^{2}\right)$ as $n \rightarrow \infty$, then for all $k \in \mathbb{N}$,

$$
U_{n} \oplus \Omega_{k} \rightarrow U \oplus \Omega_{k} \quad \text { as } n \rightarrow \infty
$$

It follows from above that $X_{f_{n}} \rightarrow X_{f}$ in $B_{1}$ and $Y_{U_{n}} \rightarrow Y_{U}$ in $B_{2}$ as $n \rightarrow \infty$. Moreover, the following can also be inferred.

Theorem 11 The mappings defined in Remark 10 are continuous embedding of $L^{1}\left(\mathbb{R}_{+}\right)$ (resp. $\left.L^{1}\left(\mathbb{R}_{+}^{2}\right)\right)$ into the space $B_{1}$ (resp. $\left.B_{2}\right)$.

\section{General properties}

In this section, we provide certain properties of the generalized natural integral transform. In fact, the results here are brief and concise, and give the reader a general overview of the generalized operator as most of similar properties are enumerated in the previous work of the author.

Theorem 12 Let $X_{U_{n}}=\left(\frac{U_{n}}{\delta_{n}}\right)$. Then the mapping $X_{U_{n}} \rightarrow Y_{U_{n}}$, defined by

$$
U_{A} X_{U_{n}}=Y_{U_{n}}
$$

is linear and coincides with the classical transform $M_{\alpha, k}: L^{1}\left(\mathbb{R}_{+}\right) \rightarrow L^{1}\left(\mathbb{R}_{+}^{2}\right)$. 
Proof Linearity is obvious. To show consistency of the transform $M_{\alpha, k}$, let $U \in L^{1}\left(\mathbb{R}_{+}\right)$, then $U$ can be identified in $B_{1}$ as $X_{U}$ where $X_{U}=\left(\frac{U \ominus \delta_{n}}{\delta_{n}}\right)$, which is the representation of $U$ in $B_{1}$. Indeed, $\left\{\delta_{1}, \delta_{2}, \ldots, \delta_{n}, \ldots\right\}$ is independent of $\left(\frac{U \ominus \delta_{n}}{\delta_{n}}\right)$. Now by the convolution theorem, we have

$$
\bar{M}_{\alpha, k} X_{U}=\left(\frac{M_{\alpha, k}\left(U \ominus \delta_{n}\right)}{\delta_{n}}\right)=\left(\frac{M_{\alpha, k} U \oplus \delta_{n}}{\delta_{n}}\right)=Y_{U} .
$$

Therefore, $Y_{U}$ is the identification in $B_{2}$ of $M_{\alpha, k} U$ in $L^{1}\left(\mathbb{R}_{+}^{2}\right)$.

The proof is therefore finished.

Theorem 13 Let $X_{f_{n}}=\left(\frac{U_{n}}{\delta_{n}}\right)$ and $\bar{M}_{\alpha, k} X_{U_{n}}=Y_{U_{n}}$. Then the mapping $X_{U_{n}} \rightarrow Y_{U_{n}}$ is one-toone, onto, and continuous with respect to the convergence of the Boehmian spaces. A similar proof for this theorem can be performed by a similar way to that of $[27,28]$. Hence it has been omitted.

We introduce the inverse integral operator of $U_{A}$ as follows.

Definition 14 Let $Y_{U_{n}} \in B_{2}, Y_{U_{n}}=\bar{M}_{\alpha, k} X_{U_{n}}=\frac{M_{\alpha, k} \chi_{n}}{\delta_{n}},\left(\delta_{n}\right) \in \Delta, X_{U_{n}}=\left(\frac{U_{n}}{\delta_{n}}\right)$. We define the inverse $\bar{M}_{\alpha, k}$ integral operator of $Y_{U_{n}}$ as

$$
\bar{M}_{\alpha, k}^{-1} Y_{U_{n}}=X_{U_{n}} .
$$

Theorem 15 The inverse mapping $Y_{U_{n}} \rightarrow X_{U_{n}}$ is linear.

Proof Consider two Boehmians $Y_{V_{n}}$ and $Y_{U_{n}}$ in $B_{2}$, where $Y_{V_{n}}=\left(\frac{M_{\alpha, k} V_{n}}{\delta_{n}}\right)$ and $Y_{U_{n}}=$ $\left(\frac{M_{\alpha, k} U_{n}}{\epsilon_{n}}\right)$. Then, for all $n \in \mathbb{N}$, the convolution theorem and the linearity of the integral reveal

$$
Y_{V_{n}}+Y_{U_{n}}=\left(\frac{M_{\alpha, k} V_{n} \oplus \epsilon_{n}+M_{\alpha, k} U_{n} \oplus \epsilon_{n}}{\delta_{n} \ominus \epsilon_{n}}\right)=\left(\frac{M_{\alpha, k}\left(V_{n} \ominus \epsilon_{n}+U_{n} \ominus \delta_{n}\right)}{\delta_{n} \ominus \epsilon_{n}}\right) .
$$

Hence, Definition 14 yields

$$
\bar{M}_{\alpha, k}^{-1}\left(Y_{V_{n}}+Y_{U_{n}}\right)=\left(\frac{V_{n} \ominus \epsilon_{n}+U_{n} \ominus \delta_{n}}{\delta_{n} \ominus \epsilon_{n}}\right) .
$$

Notion of addition in $B_{1}$ implies

$$
\bar{M}_{\alpha, k}^{-1}\left(Y_{V_{n}}+Y_{U_{n}}\right)=X_{V_{n}}+X_{U_{n}}
$$

where $X_{V_{n}}=\left(\frac{V_{n}}{\delta_{n}}\right)$ and $X_{U_{n}}=\left(\frac{U_{n}}{\epsilon_{n}}\right)$. To complete the proof of the theorem, we indeed, for some $\eta \in \mathbb{C}$ and all $n \in N$, have

$$
\bar{M}_{\alpha, k}^{-1}\left(\eta Y_{V_{n}}\right)=\eta \bar{M}_{\alpha, k}^{-1} Y_{V_{n}}
$$

This finishes the proof of the theorem.

The generalized convolution theorem can be drawn as follows. 
Theorem 16 Let $Y_{U_{n}} \in B_{2}$ and $U \in D$. Then we have

(i) $\bar{M}_{\alpha, k}^{-1}\left(Y_{U_{n}} \oplus U\right)=X_{U_{n}} \ominus U$,

(ii) $\bar{M}_{\alpha, k}\left(X_{U_{n}} \ominus U\right)=Y_{U_{n}} \oplus U$.

Proof Assume $Y_{U_{n}} \in B_{2}$ and $U \in D$. Then we have

$$
\bar{M}_{\alpha, k}^{-1}\left(Y_{U_{n}} \oplus U\right)=\bar{M}_{\alpha, k}^{-1}\left(\frac{M_{\alpha, k} U_{n}}{\epsilon_{n}} \oplus U\right)=\bar{M}_{\alpha, k}^{-1}\left(\frac{M_{\alpha, k} U_{n} \oplus U}{\epsilon_{n}}\right)
$$

By using Theorem 2 and Eq. (13), the above equation reveals

$$
\bar{M}_{\alpha, k}^{-1}\left(Y_{U_{n}} \ominus U\right)=\bar{M}_{\alpha, k}^{-1}\left(\frac{M_{\alpha, k}\left(U_{n} \ominus U\right)}{\epsilon_{n}}\right)=\left(\frac{U_{n}}{\epsilon_{n}} \ominus U\right)=X_{U_{n}} \ominus U
$$

Proof of the part $\bar{M}_{\alpha, k}\left(X_{U_{n}} \ominus U\right)=Y_{U_{n}} \oplus U$ is quite similar.

This finishes the proof of the theorem.

\section{Acknowledgements}

The authors would like to thank their friends and Springer Nature for their support.

\section{Funding}

No funding sources to be declared.

\section{Availability of data and materials}

Please contact the author for data requests.

\section{Competing interests}

The authors declare that they have no competing interests.

\section{Authors' contributions}

The authors contributed equally and significantly in writing this paper. All authors read and approved the final manuscript.

\section{Author details}

'Department of Physics and Basic Sciences, Faculty of Engineering Technology, Al-Balqa Applied University, 11134 Amman, Jordan. ${ }^{2}$ Department of Economics Faculty of Economics, Administrative and Social Sciences, Hasan Kalyoncu University, TR-27410 Gaziantep, Turkey.

\section{Publisher's Note}

Springer Nature remains neutral with regard to jurisdictional claims in published maps and institutional affiliations.

Received: 1 February 2021 Accepted: 2 March 2021 Published online: 10 March 2021

\section{References}

1. Srivastava, H.M., Luo, M., Raina, R.K.: A new integral transform and its applications. Acta Math. Sci. 35B(6), 1386-1400 (2015)

2. Khan, Z.H., Khan, W.A.: N-transform properties and applications. NUST J. Eng. Sci. 1(1), 127-133 (2008)

3. Belgacem, F.B.M., Silambarasan, R.: Maxwell's equations solutions by means of the natural transform. Math. Eng. Sci. Aerosp. 3(3), 313-323 (2012)

4. Al-Omari, S.K.Q.: Natural transform in boehmian spaces. Nonlinear Stud. 22(2), 291-297 (2015)

5. Belgacem, F.B.M., Silambarasan, R.: Applications of the natural transform to Maxwell equations. In: Prog. Electromag. Resear. Symp. Proc., Suzhou, pp. 12-16 (2011)

6. Belgacem, F.B.M., Silambarasan, R.: Theory of natural transform. Math. Eng. Sci. Aerosp. 3, 105-135 (2012)

7. Al-Omari, S.K.Q., Agarwal, P.: Some general properties of a fractional Sumudu transform in the class of Boehmians. Kuwait J. Sci. 43(2), 206-220 (2016)

8. Al-Omari, S.K.Q.: Certain results related to the $\mathrm{N}$-transform of certain class of functions and differential operators. Adv. Differ. Equ. 2018, 7 (2018)

9. Srivastava, H.M.: Some remarks on a generalization of the Stieltjes transform. Publ. Math. (Debr.) 23, 119-122 (1976)

10. Feng, S., Zhigang, P.: The inverse Laplace transform theorem of Henstock-Kurzweil integrable function. Acta Math. Sci. 27A(6), 1155-1163 (2007)

11. Atangana, A., Kilicman, A.: The use of Sumudu transform for solving certain nonlinear fractional heat-like equations. Abstr. Appl. Anal. 2013, Article ID 737481 (2013) 
12. Kilicman, A., Gadain, H.E.: On the applications of Laplace and Sumudu transforms. J. Franklin Inst. 247, 848-862 (2010)

13. Singh, J., Kumar, D., Kilicman, A.: Homotopy perturbation method for fractional gas dynamics equation using Sumudu transform. Abstr. Appl. Anal. 2013, Article ID 934060 (2013)

14. Al-Omari, S.K.Q., Kilicman, A.: An estimate of Sumudu transform for Boehmians. Adv. Differ. Equ. 77, 1-12 (2013)

15. Al-Omari, S.K.Q.: Boehmian spaces for a class of Whittaker integral transformations. Kuwait J. Sci. 43(4), 32-38 (2016)

16. Eltayeb, H., Kilicman, A.: A note on the Sumudu transforms and differential equations. Appl. Math. Sci. 4(22), 1089-1098 (2010)

17. Kilicman, A., Eltayeb, H., Agarwal, R.P.: On Sumudu transform and system of differential equations. Abstr. Appl. Anal. 2010, Article ID 598702 (2010)

18. Zemanian, A.H.: Generalized Integral Transformation. Dover, New York (1968) First Published by Interscience Publishers

19. Pathak, R.S.: Integral Transforms of Generalized Functions and Their Applications. Gordon \& Breach, New York (1997)

20. Banerji, P., Al-Omari, K., Debnath, L.: Tempered distributional Fourier sine (cosine) transform. Integral Transforms Spec. Funct. 17(11), 759-768 (2006)

21. Al-Omari, S.K.Q., Agarwal, P., Choi, J.: Real covering of the generalized Hankel-Clifford transform of Fox kernel type of a class of Boehmians. Bull. Korean Math. Soc. 52(5), 1607-1619 (2015)

22. Boehme, T.K.: The support of Mikusinski operators. Trans. Am. Math. Soc. 176, 319-334 (1973)

23. Nemzer, D.: Periodic Boehmians II. Bull. Aust. Math. Soc. 44, 271-278 (1991)

24. Nemzer, D.: The Laplace transform on a class of Boehmians. Bull. Aust. Math. Soc. 46, 347-352 (1992)

25. Al-Omari, S.K.Q.: On the application of natural transforms. Int. J. Pure Appl. Math. 84, 729-744 (2013)

26. Karunakaran, V., Vembu, R.: Hilbert transform on periodic Boehmians. Houst. J. Math. 29, 439-454 (2005)

27. Al-Omari, S.K.Q., Baleanu, D.: Some remarks on short-time Fourier integral operators and classes of rapidly decaying functions. Math. Methods Appl. Sci. 42(16), 5354-5361 (2018)

28. Al-Omari, S., Baleanu, D.: Quaternion Fourier integral operators for spaces of generalized quaternions. Math. Methods Appl. Sci. 41, 9477-9484 (2018)

29. Al-Omari, S.K.Q.: Some estimate of a generalized Bessel-Struve transform on certain space of generalized functions. Ukr. Math. J. 69(9), 1155-1165 (2017)

30. Al-Omari, S.K.Q.: Some characteristics of S transforms in a class of rapidly decreasing Boehmians. J. Pseudo-Differ. Oper. Appl. 5(4), 527-537 (2014)

31. Al-Omari, S.: On q-analogues of the natural transform of certain q-Bessel functions and some application. Filomat 31(9), 2587-2598 (2017)

32. Mikusinski, P.: Boehmians and pseudoquotients. Appl. Math. Inf. Sci. 5, 192-204 (2011)

33. Al-Omari, S.K.Q.: On the generalized double Sumudu transforms of distributions and space of Boehmians. Int. J. Algebra 6(3), 139-146 (2012)

34. Al-Omari, S.K.Q., Al-Omari, J.F.: Some extensions of a certain integral transform to a quotient space of generalized functions. Open Math. 13, 816-825 (2015)

\section{Submit your manuscript to a SpringerOpen ${ }^{\circ}$ journal and benefit from:}

- Convenient online submission

- Rigorous peer review

- Open access: articles freely available online

- High visibility within the field

Retaining the copyright to your article

Submit your next manuscript at springeropen.com 